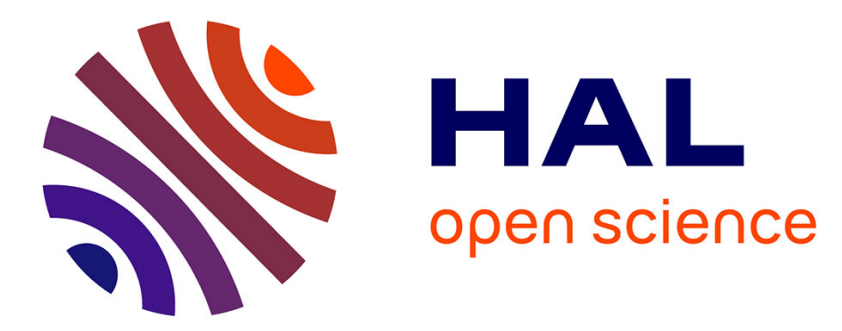

\title{
Motion of Optically Heated Spheres at the Water-Air Interface
}

\author{
A. Girot, N. Danné, Alois Würger, Thomas Bickel, Kuan Fang Ren, J. C. \\ Loudet, B. Pouligny
}

\section{To cite this version:}

A. Girot, N. Danné, Alois Würger, Thomas Bickel, Kuan Fang Ren, et al.. Motion of Optically Heated Spheres at the Water-Air Interface. Langmuir, 2016, 32 (11), pp.2687 - 2697. 10.1021/acs.langmuir.6b00181 . hal-01294298

\section{HAL Id: hal-01294298 \\ https://hal.science/hal-01294298}

Submitted on 17 Jan 2018

HAL is a multi-disciplinary open access archive for the deposit and dissemination of scientific research documents, whether they are published or not. The documents may come from teaching and research institutions in France or abroad, or from public or private research centers.
L'archive ouverte pluridisciplinaire HAL, est destinée au dépôt et à la diffusion de documents scientifiques de niveau recherche, publiés ou non, émanant des établissements d'enseignement et de recherche français ou étrangers, des laboratoires publics ou privés.

\section{(ㄷ)(1) $\$$}

Distributed under a Creative Commons Attribution - NonCommerciall 4.0 International 


\title{
Motion of Optically Heated Spheres at the Water-Air Interface
}

\author{
A. Girot, ${ }^{\dagger, \ddagger}$ N. Danné, ${ }^{\dagger, \ddagger}$ A. Würger, ${ }^{\ddagger}$ T. Bickel, ${ }^{\ddagger}$ F. Ren, ${ }^{\S}$ J. C. Loudet, ${ }^{\dagger}$ and B. Pouligny ${ }^{*}, \dagger$ \\ ${ }^{\dagger}$ Université de Bordeaux, Centre de recherche Paul Pascal (CRPP), 33600 Pessac, France \\ ${ }^{\ddagger}$ Université de Bordeaux, Laboratoire Ondes et Matière d'Aquitaine (LOMA), 33400 Talence, France \\ ${ }^{\S}$ CORIA UMR6614, Normandie Université, CNRS, Université et INSA de Rouen, Avenue de l’Université, 76800 Saint Etienne du \\ Rouvray, France
}

\begin{abstract}
A micrometer sized spherical particle classically equilibrates at the water-air interface in partial wetting configuration, causing about no deformation to the interface. In condition of thermal equilibrium, the particle just undergoes faint Brownian motion, well visible under a microscope. We report experimental observations when the particle is made of a light absorbing material and is heated up by a vertical laser beam. We show that, at small laser power, the particle is trapped in on axis configuration, similarly to 2 dimensional trapping of a transparent sphere by optical forces. Conversely, on axis trapping becomes unstable at higher power. The particle escapes off the laser axis and starts orbiting around the axis. We show that the laser heated particle behaves as a microswimmer with velocities on the order of several 100 $\mu \mathrm{m} / \mathrm{s}$ with just a few milliwatts of laser power.
\end{abstract}

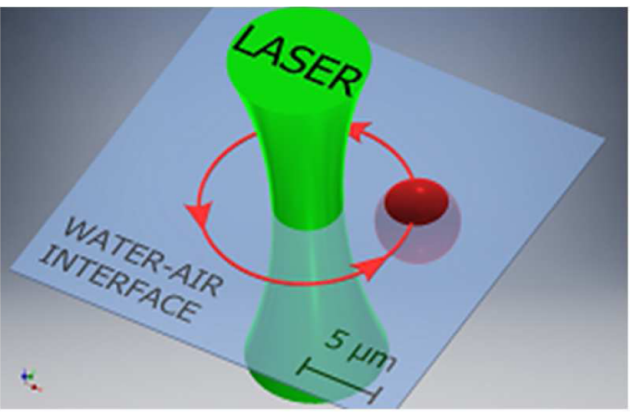

\section{INTRODUCTION}

Laser beams may be used to manipulate micrometer sized particles, using optical forces. A very simple experiment, called "optical levitation", ${ }^{1}$ can be carried out with just a transparent sphere in water, and a moderately focused laser beam, vertically oriented. The particle, initially sitting on the floor of a glass cuvette, responds to the laser beam by self centering, and, if the power is high enough, it lifts up under the action of radiation pressure. The motion stops when the particle hits the top wall (ceiling) of the cuvette. The configuration is a case of two dimensional (2D) trapping, because the particle stands about still with its center on the beam axis. Only a faint Brownian motion is perceptible, which makes the particle slightly wander around the beam axis. As well known, it is possible to produce three dimensional (3D) trapping of the spherical particle inside bulk water using either a couple of moderately focused beams, ${ }^{2-6}$ or a single tightly focused beam, as in optical tweezers (OT).

Figure 1 shows a case of simple optical levitation. Here the cuvette is only partly filled with water, meaning that the top boundary now is a water-air (WA) interface. The levitation force pushes the particle against the interface. In general, the particle partly crosses the interface and ends in partial wetting configuration (Figure 1b). If the sphere surface is ideally smooth and chemically uniform, the three phase contact line is a circle corresponding to the prescribed value of the contact angle for the material of interest. As a micrometer sized particle has a negligible weight on the scale of surface forces, the interface remains flat and horizontal. ${ }^{8}$ In this experiment, the sphere still self centers on the beam axis, not differently from the former situation with a water-glass interface.
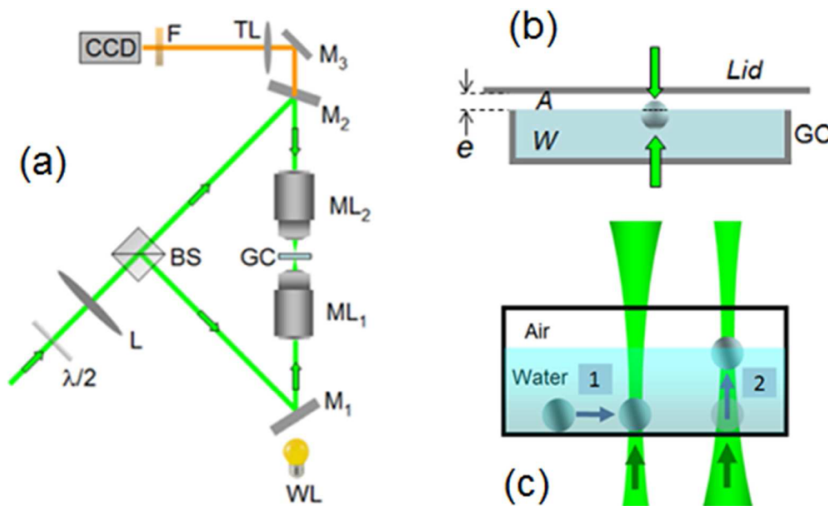

Figure 1. (a) Optical setup. The setup functions as a two beam levitator, including a classical microscope (TL: tube lens) with bright field illumination (WL: white light Koehler source). $\mathrm{M}_{1,2}$ : dichroic mirrors. BS: polarizing beam splitter. $\lambda / 2$ : half wave $(514 \mathrm{~nm})$ plate. The laser beamwaist (b.w.) is located within the focal plane common to $\mathrm{ML}_{1,2}$. The b.w. radius $\omega_{0}$ can be varied by changing lens $\mathrm{L}$ and/or the condenser $\left(\mathrm{ML}_{2}\right)$. (b) Close up view of the sample (GC: glass cuvette) between microscope objectives $\left(\mathrm{ML}_{1,2}\right)$. The green arrows in panel $\mathrm{b}$ are symbols for the up and down beams. (c) The experimental procedure starts with capturing a particle (1), followed by levitation (2) up to the WA interface. Distances are not to scale, for clarity.

In this paper, we deal with spheres that are only partially transparent; then a part of the light from the laser beam is 
absorbed by the particle material and dissipated into heat. Conversely to transparent particles, strongly absorbing spherical particles cannot be trapped on the axis of a low aperture Gaussian beam. In this case, optical forces just push the particle out of the beam axis. ${ }^{9}$ We are interested in moderately absorbing particles, meaning that absorption is small enough to avoid rejection of the particle from the beam. Our particles can still be trapped with a Gaussian beam, at least at small laser power $(P)$. In terms of trapping configuration, heating does not make a great difference from the case of a transparent particle, insofar as the particle is entirely in water. The true difference comes when the particle sits across the WA interface. As we report below, the particle is pushed out of the beam and undergoes different types of motion, sometimes with velocities on the order of several $100 \mu \mathrm{m} / \mathrm{s}$.

The phenomena of interest are definitely related to heating of the particle and to heat transfer to the surrounding WA interface. Prior to this work, different studies have been devoted to the effects of laser illumination on fluid interfaces. These include direct heating through light absorption by the fluid, ${ }^{10-13}$ or photochemical transformation of the inter face. $^{14,15}$ In these works, a layer of surface active molecules was present on the interface, and the reported phenomena were due to responses of the surfactant films, either directly to the laser induced temperature profile or to the photochemical transformation of the film within the illuminated area. In our case, the interface is pure water-air (though contamination may play a role, as we will comment), and the heat source is localized within the body of the spherical particle. We are mainly interested in the reaction of the particle to the flow that it has itself created.

In the limit when the beam cross section is much wider than the particle diameter $(2 a)$, the system may be viewed as simply a hot sphere sitting on a WA interface. In the latter limit, light only acts as the fuel needed to keep the particle at higher temperature than the water bath far from it. The configuration is then equivalent to that of a particle that might be hot by itself, as if it were made of a radioactive material, or if heat were produced by some internal chemical reaction. In our case, the fuel that provides power is laser light. Contrary to chemical reactants, light is an endless fuel. As we will see, the hot sphere self propels on the interface at high velocity, realizing a case of a microswimmer confined to a fluid surface. ${ }^{16}$

The paper is organized as follows. The information about particles and the optical setup is given in Section 2. Observations regarding the motions of the particles are reported in Section 3: we start with the responses of particles in bulk water, to gather basic information about optical forces. We then turn to the behavior at the WA interface, for different beam sizes and powers. These data are complemented in Section 4 with observations about the flow around the hot particle, using tracers. The information from experiments is analyzed and discussed in Section 5. The goal there is to spot what we believe to be the main mechanisms at work in the particle propulsion. The paper ends with conclusions and prospects.

\section{MATERIALS AND METHODS}

In our experiments we use a green laser (Coherent Genesis) at wavelength $514 \mathrm{~nm}$, as sketched in Figure 1 . As a basic requirement, we needed particles that absorb green light, and, most importantly, that are not degraded under laser illumination. The latter requirement made us eliminate dye doped fluorescent particles, because they would

systematically bleach under few milliwatt illumination. We used magnetic spheres ("magspheres"), about $5 \mu \mathrm{m}$ in diameter. Such particles (provided by Spherotec) have a transparent PS spherical core, inside a PS shell filled with iron oxide crystallites (see sketch in Figure $2 \mathrm{a})$. The magnetic properties of such particles were not exploited in

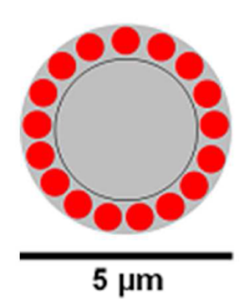

(a)

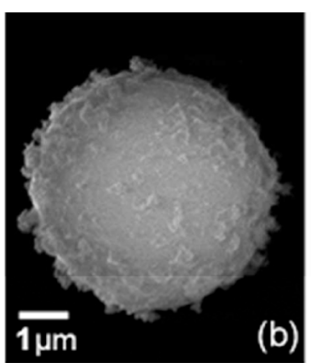

Figure 2. (a) Scheme of the structure of a magsphere. Red spots are symbols for iron oxide inclusions. (b) Electron microscope image of the particle rough surface.

our experiments. The interest of magspheres resides in the absorption, together with high resistance to photodegradation, of the iron oxide inclusions. Indeed, these particles showed no sign of degradation in the experiments reported throughout this article. Degradation only occurred when the particle was held static and centered on the laser beam at high power, corresponding to intensities $\geq 0.24 \mathrm{~mW} /(\mu \mathrm{m})^{2}$ (see Section 4). The spheres looked about spherical within the resolution of optical microscope, but scanning electron microscopy revealed that their surfaces were rough, with protrusions up to $0.4 \mu \mathrm{m}$ in amplitude (Figure 2b).

Samples consist of very dilute suspensions of magspheres in water, inside a circular quartz cuvette with an open top (a kind of small Petri dish; internal dimensions: $22 \mathrm{~mm}$ in diameter, $3 \mathrm{~mm}$ in height; see Figure $1 \mathrm{~b}$ ). Prior to each experiment, the cuvette was cleaned by $24 \mathrm{~h}$ immersion within sulfochromic acid and rinsed with Millipore Milli Q water. The edge of the cuvette has been kept sharp, to provide contact line pinning. In the standard procedure, we overfill the cuvette and remove a small volume of water. Contact line pinning allows us to obtain a flat WA interface. The cuvette is covered with a circular glass lid, to limit the pollution of the interface by atmospheric contaminants, massive water evaporation, and agitation caused by air turbulence. The lid lies on spacers to keep a small air gap $(e)$ above the WA interface (Figure 1b). Not surprisingly, a large gap does not provide enough protection, as too much air can sweep the interface. Closing the system $(e=0)$ is not acceptable either, because of heavy condensation of the water vapor on the glass. We found a satisfactory trade off with $e \approx 0.3$ $\mathrm{mm}$.

In our setup, the vertical laser beam is focused inside the sample by means of a long working distance objective $\left(M L_{1}\right.$ in Figure 1a). The beamwaist radius $\omega_{0}$ can be varied from 1.3 to about $33 \mu \mathrm{m}$, using different types of microscope objectives $(50,20,10,2.5 \mathrm{X})$ and lenses of different focal lengths upstream of the objectives (before BS; see Figure 1a). The laser beam allows us to pick up a single particle in bulk suspension, and levitate it up to the WA interface (Figure 1c). Optical levitation is made possible by the radiation pressure from the laser beam. Powers on the order of milliwatts provide forces in the piconewton range, well enough to make a particle transit from the bottom of the cuvette up to the WA interface within about $2 \mathrm{mn}$, in standard operation.

In general, the levitated particle locks to the interface in partial wetting configuration (Figure 1b). Locking can be verified by switching off the laser. The particle then slowly drifts off due to Brownian motion and parasitic interfacial convection, but keeps in focus within the microscope image. Conversely, a particle in contact but not locked to the interface, then still in complete wetting configuration, detaches from the interface within less than a minute and gets blurred. Though we may ascertain that a particle sits across the interface, the microscope does not have enough resolution to 
provide accurate images of a contact line on the particle surface. In a few examples, though, faint images of contact lines could be obtained, and we could see that their shapes definitely departed from a circle, as it should have been on a perfect sphere. This observation was not a surprise because the roughness of the particles' surfaces (Figure 2b) inevitably promotes contact line pinning. Note that contact lines on polystyrene particles in general have irregular shapes, as was mentioned in previous works, ${ }^{17,18}$ due to surface roughness and chemical heterogeneity. ${ }^{19}$ Irregular contact lines are out of equilibrium features that evolve through thermal activation, increasing the effective contact angle in time. ${ }^{17,20}$

Some of our experiments were operated with a couple of contra propagating beams, as described in refs $5,6,21$. In addition to the upward directed beam, the two beam configuration provides a downward directed beam (see Figure 1). We may switch from "up" to "down" beam, or use both beams in combination, with any power repartition. Both beams have a common beamwaist plane, and can be superposed with submicrometer accuracy.

\section{OBSERVATIONS}

Optical Levitation of Magspheres in Bulk Water. The magspheres are heavier than water and, consequently, are easily found lying on the cell bottom. In routine operation, we only use the up beam, at moderate power $(\sim 1 \mathrm{~mW})$. The sample cell is moved horizontally ( $x, y$ directions) to bring a particle close to the laser beam. The particle then starts lifting up (Figure 1c, step 2). Particle ascension within bulk water can be easily stopped by reducing the power, and the particle image can be kept in focus by adjusting the altitude of the cell. In such conditions, the particle stays in the beamwaist plane $(z=0)$ with its buoyant weight balanced by the laser induced force. Brownian motion makes the particle's position $x, y$ fluctuate around the beam axis. Excursions are large but limited $(\leq a)$, meaning that $2 \mathrm{D}$ trapping is evident but faint. The latter characteristic is not surprising, because of the smallness of typical levitation powers, much less than $1 \mathrm{~mW}$ in general. The vertical equilibrium is unstable, ${ }^{1,22,23}$ but it takes several seconds for the particle to move out of focus, either up or down. Playing with the laser power allows determining the levitation power $P_{\text {lev }}$ within a few percent. With this amount of accuracy, the particle keeps at constant $z$ for half a minute, typically. For illustration, we find $P_{\text {lev }} \approx 0.20 \mathrm{~mW}$ for $\omega_{0}=7.3$ $\mu \mathrm{m}$, and $P_{\mathrm{lev}} \approx 0.045 \mathrm{~mW}$ for $\omega_{0}=3.6 \mu \mathrm{m}$, with a particle of radius $a \approx 2.2 \mu \mathrm{m}$. These values may vary by $\pm 30 \%$ among experiments carried out with different particles.

Once the particle is levitated, the laser may be switched off. The particle then slowly moves down back to the cell bottom. This allows us to measure the particle sedimentation velocity:

$$
v_{\text {sed }}=\frac{2}{9} \frac{\Delta \bar{\rho} g a^{2}}{\eta}
$$

and the particle's buoyant weight $\bar{m} g=6 \pi \eta a v_{\text {sed }}$. In eq 1 , $\Delta \bar{\rho}=\bar{\rho}-\rho_{\mathrm{W}}$ is the difference between the particle average density and that of water, and $g$ is the gravity acceleration. $\nu_{\text {sed }}$ is found $\approx 1 \mu \mathrm{m} \cdot \mathrm{s}^{-1}$, from which we estimate $\bar{m} g \approx 0.06 \mathrm{pN}$ and $\Delta \bar{\rho} \approx 0.13 \pm 0.01 \mathrm{~g} \cdot \mathrm{cm}^{-3}$. From the value of $\Delta \bar{\rho}$ we can estimate the volume fraction of the iron oxide inside the particle shell and deduce values for the complex index of refraction used in the model for optical forces calculation (see Section 5).

Particle Behavior at WA Interface. In the levitation experiment, a transparent PS particle keeps about centered on the beam axis, in bulk water. Centering is faintly perturbed by the particle's Brownian motion, on the scale of a micrometer in typical conditions. Perturbations become much more intense when the particle comes close to the WA interface, but the particle can still be maintained within the beam and pushed against the interface. Transition to the partial wetting configuration happens within a delay, which is very variable between different experiments, from seemingly immediate to several minutes. When the transition is achieved, meaning that the particle sits across the interface, it can still be trapped (in 2D), but perturbations may be large enough for the particle to escape off the beam. These perturbations are due to disordered motions along the interface, with velocities that may be up to a few micrometers per second in our conditions. Mechanical vibrations are obviously a cause of vibration of the interface. Another cause of parasitic motions is the "interfacial turbulence", which can be revealed by infrared imaging. ${ }^{24}$ Though these perturbations are not directly observable in visible light, their effects are readily sensed in optical manipulation experiments.

Our experience is that the turbulence is very intense (meaning that the corresponding hydrodynamic drag is large enough to compete with $\mathrm{pN}$ forces from radiation pressure) with freshly prepared samples in a quartz cuvette. Aging of the system gets signaled by a decrease of the interface turbulence. The evolution is visible within less than an hour. The interface progressively becomes rigid, evidencing contamination by surface active species. ${ }^{13,25,26}$ Contamination may happen within minutes in some cases, especially when other materials (e.g., a cuvette made of pyrex glass) are used, or when less care has been taken in cleaning the glassware.

Magspheres. Magspheres behave similarly to PS particles until contact to the interface. A magsphere usually pierces the interface and transits to partial wetting within seconds. At low power (less than $1 \mathrm{~mW}$, roughly), the particle is attracted toward the beam axis, in the same way as the PS particle, but the configuration is very sensitive to perturbations. The magsphere keeps within a radius $(a \approx 2.2 \mu \mathrm{m})$ of the beam axis provided that interfacial turbulence in the region of interest is weak. An example of $2 \mathrm{D}$ trapping of a magsphere at WA interface, for $\omega_{0}=3.5 \mu \mathrm{m}$, is shown in the Supporting Information Video S1. Note that attraction to the beam axis at low power is evident only when $\omega_{0}$ is not too large compared to a. Not surprisingly, very wide beams $\left(\omega_{0} \gg a\right)$ do not produce 2D trapping.

The main difference between the transparent particle and the magsphere comes when the laser power is increased. Observations were made for different values of the beamwaist, from smaller $\left(\omega_{0}=1.48 \mu \mathrm{m}\right)$ up to much larger $\left(\omega_{0}=33 \mu \mathrm{m}\right)$ than the particle radius. In moderate focusing condition $(1.4 \leq$ $\omega_{0} \leq 9 \mu \mathrm{m}$ ), we observed a transition from attraction to expulsion for powers slightly above $1 \mathrm{~mW}$. The latter value is rough, because the transition was blurred by the parasitic interfacial convection; then it is impossible to prove the existence of a sharp threshold in power, with a corresponding accurate value. Nevertheless, the transition occurred between 1 and $3 \mathrm{~mW}$ for all beamwaists within the explored range.

The experiment can be carried out with very large values of the beamwaist as well. In this case, lateral trapping does not occur, either in bulk or at the interface. However, levitation in bulk remains straightforward, insofar as we can play with the horizontal $(x, y)$ position of the cell to keep the particle close to the beam axis $(O)$, through a few manual corrections. Once the particle sits across the interface, we switch off the beam, bring the particle close to $O$, and switch the laser back on. Expulsion 
away from the axis follows quite evidently, similarly to the case of smaller beamwaists. The phenomenon is shown in Figure 3.

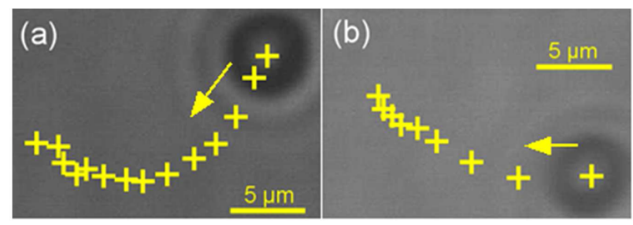

Figure 3. Magsphere at WA interface. Repulsion from laser beam axis. Laser beamwaist $\omega_{0}=33 \mu \mathrm{m}$. The photos show the particle at initial position (close to the laser beam axis), when the laser is switched on, and the arrows indicate the direction of the particle's motion. Crosses mark the positions of the particle in time, starting from laser switch on. Laser power: $16 \mathrm{~mW}$ (a), $36 \mathrm{~mW}$ (b). Time interval between successive positions: $0.39 \mathrm{~s}(\mathrm{a}), 0.10 \mathrm{~s}(\mathrm{~b})$.

Velocities are on the order of a few $\mu \mathrm{m} / \mathrm{s}$, up to a few $10 \mu \mathrm{m} / \mathrm{s}$ (see Figure $3 \mathrm{~b}$ ). Trajectories are clearly directed along a given direction, and then are well discernible from Brownian noise and interfacial perturbations. Note that the large beamwaist limit $\left(\omega_{0} \gg a\right)$ is an approximation of the academic situation of a hot sphere lying on an infinite interface, as we mentioned in the Introduction.

Orbital Motion. Moving off the beam axis is the first step in the particle's response to the laser. In general, the particle does not stop at finite distance from the axis. The motion goes on with the particle orbiting around the beam axis. The phenomenon is illustrated below in Figure $4 \mathrm{a}$ and shown in

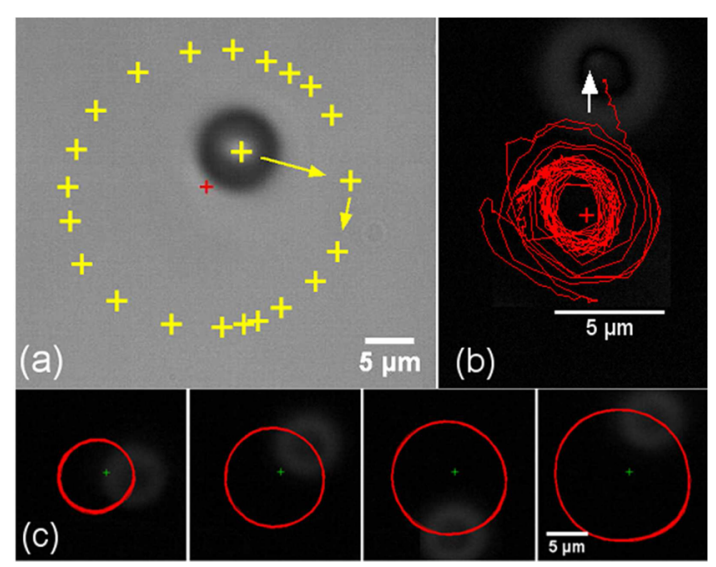

Figure 4. (a) Injection of a magsphere into a quasi circular orbit. The beam axis is marked by a red cross. $P=20 \mathrm{~mW}$ (down beam); $\omega_{0}=6.3$ $\mu \mathrm{m}$. Yellow crosses mark the positions of the particle in time, starting from laser switch on. Time interval between successive positions: $0.033 \mathrm{~s}$. The arrows indicate the initial motion off the beam axis (large arrow), and the development of a quasi circular orbit (small arrow). (b) Example of a disordered trajectory. $\omega_{0}=6.7 \mu \mathrm{m}, P=7.5 \mathrm{~mW}$. Ultimately, the particle escapes from the laser beam (white arrow). (c) Particle's quasi circular orbits around the laser beam axis (marked by a green cross). $\omega_{0}=4.3 \mu \mathrm{m}$. The orbit radius increases with the laser power. $P=3.2,7.0,10.1,19.6 \mathrm{~mW}$, from left to right.

Video S2 of the Supporting Information. In this case $\left(\omega_{0}=6.3\right.$ $\mu \mathrm{m}, P=20 \mathrm{~mW})$, the orbit is close to circular, with a radius $r_{\text {orb }}$ $\approx 11 \mu \mathrm{m}$. While the tendency for the particle to orbit around the laser axis is evident in general, "clean" circular orbits are not realized in all experiments. We frequently observed that the particle would undergo disordered motion, with loops of variable size, separated by inversions in the direction of rotation, and that the particle would ultimately escape far off the beam. An example of such behavior is shown in Figure $4 b$ and in Supporting Information Video S3. Producing clean orbits is not really within control of the experimenter in the current procedure. At least, our experience is that stable quasi circular orbits were obtained only when greatest care was taken in preparing the samples. Interface contamination presumably plays a key role in producing chaotic trajectories.

A few more examples of quasi circular orbits are displayed in Figure 4c. The sequence shows that the size of the orbit increases with the laser power $(P)$, for a given value of $\omega_{0}$. We found that $r_{\text {orb }}$ increases with both $P$ and $\omega_{0}$, as shown in Figure 5. Here $r_{\text {orb }}$ has been measured as the average radius of the not exactly circular orbit, in each case. The graphs indicate that the laser heated particle gets trapped into a finite size orbit only when $P$ is larger than a minimum value $P_{\min }$, with $1 \leq P_{\min } \leq 3$ mW. When $P<P_{\min }$, the particle is attracted toward the beam axis, as we explained above. For each value of the beamwaist, the orbit radius increases sharply starting from $P_{\min }$, and seemingly tends to saturation at large power. Data displayed in Figure 5 were obtained with a new particle each time the value of $\omega_{0}$ was changed. For each value of $\omega_{0}$, we checked that the orbit size was repeatable upon switching off and on the laser. We thus conclude that the behaviors shown in Figure 5 are robust in providing size characteristics of the orbits (of course in the context when the state of the interface allows for the existence of stable quasi circular orbits). Note that laser intensities given by $I=2 P / \pi \omega_{0}^{2}$ may seem definitely larger than the maximum admissible value $I_{\max }$ mentioned in Section 2 $\left(\approx 0.24 \mathrm{~mW} / \mu \mathrm{m}^{2}\right)$. However, orbital motions occur well off the laser beam axis; and the power intercepted by the particle, in all cases, is small such that the corresponding intensity is well within $I_{\max }$.

While the orbit radius turns out to be predictable for given characteristics of the laser beam, the azimuthal velocity $\Omega$ of the particle is not. Variations of $\Omega$ along a single period are visible in Figure $4 a$, as they are signed by fluctuations of the interval between two successive crosses. In the latter example, fluctuations were moderate. In numerous realizations, the particle intermittently went to a stop and, rather surprisingly, $\Omega$ even reversed in sign, without changing the orbit radius. Measurements of $\Omega$ (in absolute value), corresponding to the same experiments as in Figure 5a, are displayed in Figure 5b. There is no readable tendency, as $|\Omega|$ may take on values between 0 and more than $100 \mathrm{rad} / \mathrm{s}$. $|\Omega|$ values look randomly distributed. Note that curvilinear velocities $\nu=\Omega r_{\text {orb }}$ may be quite large, up to almost $1 \mathrm{~mm} / \mathrm{s}$.

\section{FLOW VISUALIZATION}

Optical forces are not able to produce particle motion at such high velocities (see the discussion, Section 5). It is then rather obvious that heat from the particle generates a flow, which in turn drives the motion of the magsphere. Below we report information about this flow, which we obtained by observing motions of tracer particles around a laser heated magsphere.

Tracers were submicrometer PS particles $(0.77 \mu \mathrm{m}$ in diameter), which were in suspension in bulk water. We made a few attempts with tracers located at the WA interface, by spreading from a suspension in a water/propanol mixture (1:1 vol.). However, the latter procedure was abandoned, because it would cause rigidifying of the interface. The bulk suspension did not pose this problem and was about neutral vis à vis the interface, as far as we could tell from optical manipulation of 

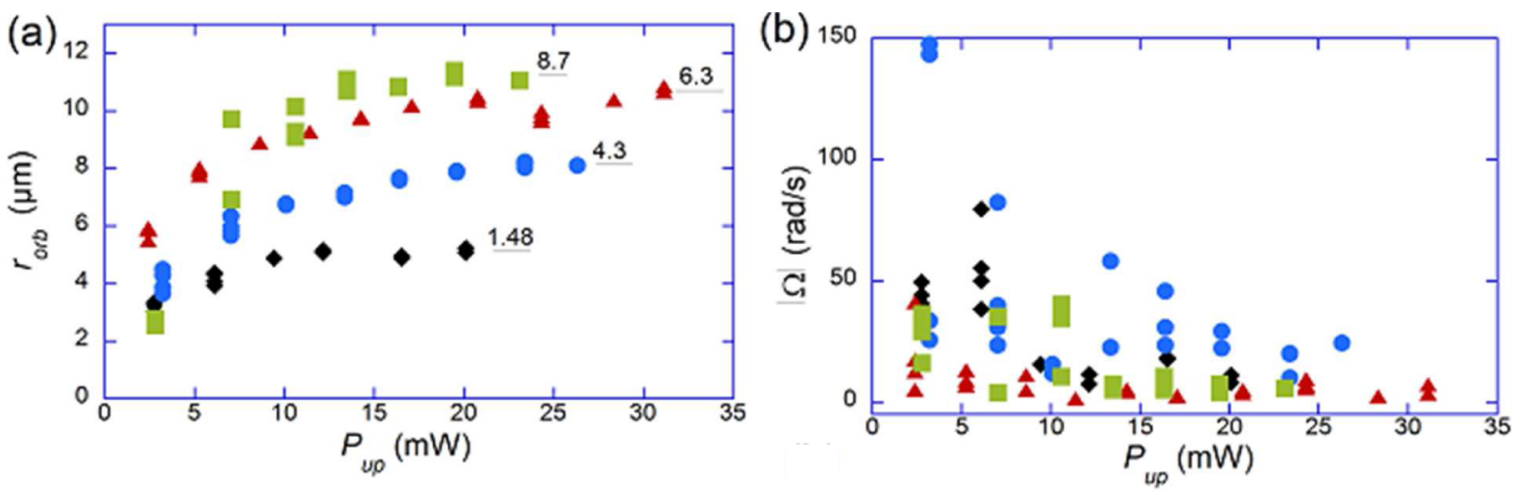

Figure 5. Influence of beam power and beamwaist radius on characteristics of quasi circular orbits. (a) Orbit radius $r_{\text {orb }}$. The beamwaist radius is indicated at right of the corresponding data, in micrometers. (b) Azimuthal velocities $\Omega$, in absolute value. Same symbols as in panel a. The figure illustrates the random character of the orbital velocity, in contrast to the orbit size. The experiments are done with the up beam.

large PS particles in the presence of the tracers. The drawback with tracers in bulk water is that they are very sparse within the zone where the main features of the flow are most visible, in fact a layer about $20 \mu \mathrm{m}$ in thickness below the interface. Nevertheless, a few streamlines could be seen in each single experiment, providing exploitable information about the flow characteristics.

To characterize the flow in simplest conditions, it is desirable to keep the particle immobile within the microscope image. The laser beam does not achieve particle trapping at the interface, as we saw, since heating makes the particle move. As an alternative to trapping, we moved the sample cell manually (in $x, y$, using translation stages) to keep the particle close to the laser axis. The procedure necessitates constantly correcting the cell position. We did not achieve better than $10 \mu \mathrm{m}$ in centering accuracy, but this was enough to visualize essential features of the flow. In the case of a weakly focused beam, the method allows maintaining the power intercepted by the particle $\left(P_{\text {int }}\right)$ at about constant level. For illustration, fluctuations in $P_{\text {int }}$ with $\omega_{0}=33 \mu \mathrm{m}$ (Figure 6) were limited to $\pm 9 \%$.

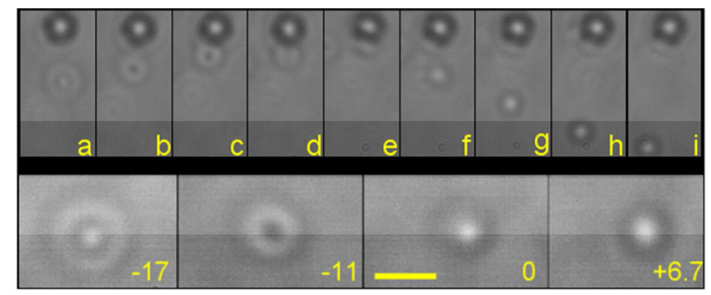

Figure 6. Attraction $(\mathrm{a}-\mathrm{e})$ of a tracer toward the laser heated magsphere and repulsion $(\mathrm{f}-\mathrm{i})$. The tracer comes from below the interface, moves up when getting close to the magsphere, and moves radially outward parallel to the interface. Time increases from left to right; interval between two successive frames $=57.3 \mathrm{~ms} . P=6.6 \mathrm{~mW}$, $\omega_{0}=33 \mu \mathrm{m}$. The chart below the sequence is used to decode the photos for the tracer's distance (indicated by a number, in $\mu \mathrm{m}$, in each panel) below the interface. Scale bar $=5 \mu \mathrm{m}$.

Examples of tracers' trajectories close to a laser heated magsphere are shown in Figure 6a-i. (see Video S4 in the Supporting Information). The photos are views from the top, and then show positions in $(x, y)$ coordinates. The microscope is focused on the magsphere, i.e., approximately at the waterair interface $(z=0)$. A typical image of a tracer is a faint spot, whose aspect is very sensitive to focusing, meaning that it depends on the altitude of the tracer. This dependence can be used to estimate the distance of the tracer to the interface. The chart below the $\mathrm{a}-\mathrm{i}$ sequence is a collection of reference images of tracers, which were obtained from an independent experiment with a tracer held at constant altitude and for variable focusing of the microscope. The chart helps reading each photo in the a-i sequence, essentially to ascertain whether the tracer is well below the interface, or close to it (within about $5 \mu \mathrm{m}$ ). Clearly, the tracer in panel a is about $15 \mu \mathrm{m}$ below the interface, while those in panels $\mathrm{g}-\mathrm{i}$ move along the interface. Thus, we can see the motions of tracers in $3 \mathrm{D}$, within a few micrometers vertical resolution.

As a general trend, tracers located below the interface are seen to converge toward the particle (centripetal motion). Convergence brings the tracer to a minimum distance $d_{\text {min }}$ from the magsphere's center, where the direction of motion reverses into repulsion (centrifuge motion). Repulsion is systematically observed when $d_{\min }$ is larger than the magsphere radius $\left(d_{\min }>\right.$ a), leaving the impression that the tracer bounces off the magsphere. The corresponding velocity is estimated to be $\sim 100$ $\mu \mathrm{m} / \mathrm{s}$.

When $d_{\min } \leq a$, the tracer comes close to the contact line, within the corner bounded by the water-air and the watersolid interfaces. In this case, the tracer may still bounce off the magsphere, as mentioned above, or be captured. "Capture" means that the tracer remains trapped along the contact line. In many experiments, tracers are seen to accumulate on the hot particle, building a crown thick enough to be visible in microscope images.

What we believe to be the geometry of the flow around the laser heated magspheres, including the capture of a tracer, is sketched in Figure 7. Note worthily, the aggregation of tracers around the magsphere contact line is a reversible effect, meaning that switching off the laser beam has the effect of releasing the captured tracers. The latter are seen to move off the magspheres, either gently by Brownian motion, or driven by centrifuge convection. An example of convective release is shown in Figure 8 and in Supporting Information Video S5. Operating the experiment with a large beamwaist (e.g., $\omega_{0}=33$ $\mu \mathrm{m})$ only produced gentle Brownian release. Convective release was evident only with more focused beams, as in the example shown in Figure $8\left(\omega_{0}=6.3 \mu \mathrm{m}\right)$. We presume that the difference between both cases is not essential and simply stems from the difference in laser power $\left(P_{\text {int }}\right)$ intercepted by the particle $\left(P_{\text {int }} \propto \omega_{0}^{-2}\right.$ when $\left.a \ll \omega_{0}\right)$. 


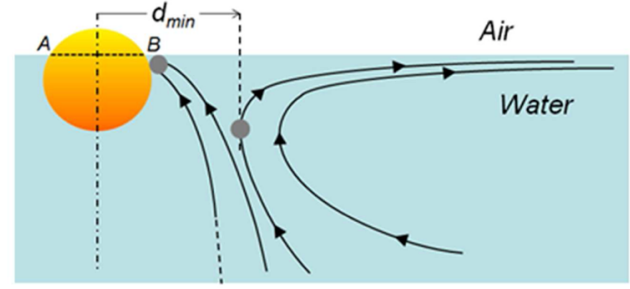

Figure 7. Geometry of the flow around the laser heated magsphere, as suggested by the observations of tracers' motions. The sketch is a vertical planar cut through the center of the magsphere (orange yellow). The laser beam, not represented, is supposed to be uniform in intensity. The toroidal flow, at right, brings tracers (gray spot) down to a minimal distance $d_{\min }$ from the magsphere's axis. The flow on the interface is centrifuge for $r \geq d_{\text {min }}$, and centripetal for $r \leq d_{\min }$. A tracer in the centripetal zone is conveyed up to the contact line $(A B)$, where it stays trapped. Switching off the laser beam releases the tracer. Dimensions are not to scale, for clarity.

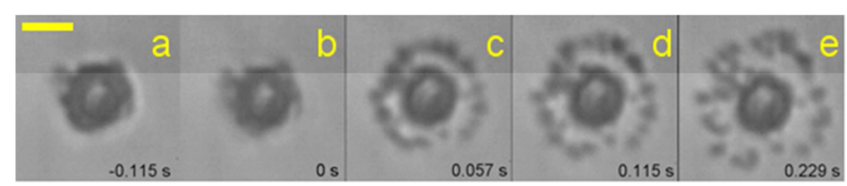

Figure 8. "Laser on-laser off" sequence showing the release of tracers that have been captured along the magspheres's contact line. Time along the sequence is indicated in each frame. When the laser is on (a), the captured tracers accumulate into a crown of irregular shape. This crown starts disintegrating once the laser is switched off (b). The tracers move off radially $(\mathrm{c}-\mathrm{e})$, indicating centrifuge convection. $\omega_{0}=$ $6.3 \mu \mathrm{m}, P=24 \mathrm{~mW}$. Scale bar $=5 \mu \mathrm{m}$.

Vortex Flows. It was not possible to systematically track tracers when a magsphere was left free to move, in particular when the particle was orbiting. Interestingly, some experiments revealed that tracers would gather into a rotating cloud that would accompany the magsphere along a circular orbit. The effect is illustrated in Figure 9 and is shown in Supporting

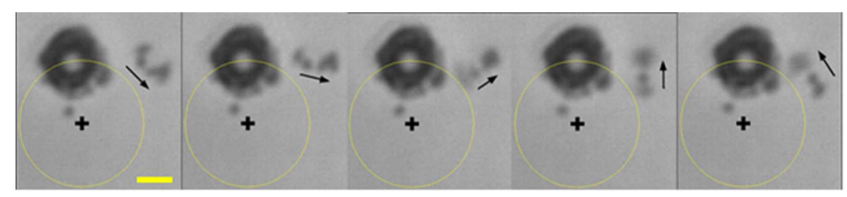

Figure 9. Tracers reveal a vortex flow ahead of an orbiting magsphere. Time increases from left to right; interval between two images $=24$ ms. The black cross marks the position of the laser beam axis. The magsphere's orbital motion (yellow circle) is clockwise, while the vortex motion (black arrows) is counterclockwise. $\omega_{0}=6.3 \mu \mathrm{m}, P=28$ $\mathrm{mW}$. Scale bar $=5 \mu \mathrm{m}$.

Information Video S6. A major vortex is visible ahead of the particle, outside of the circular orbit (yellow circle). Close inspection of the video reveals the presence of a second vortex lagging behind the particle, slightly inside the orbit. A crown of tracers keeps aggregated around the magsphere, like in Figure $8 \mathrm{a}, \mathrm{b}$.

Water-Oil Interface. As an alternative to WA interface, we made a few tests with a layer of hexadecane $(2 \mathrm{~mm}$ in thickness) above the water layer, inside a modified cell. The laser beamwaist was $\omega_{0}=6.3 \mu \mathrm{m}$. It turned out that the magspheres did not pierce the water-oil interface, contrary to the WA interface. Though the particle could be pressed by the laser pressure force (up to a few $\mathrm{pN}$ ) against the interface, it would detach and fall down within a few seconds when the laser was switched off. We noticed that the particle would self center on the laser beam axis whatever the laser power, oppositely to the observed off centering in the case of the WA interface.

Interestingly, the experiment brought indirect information about the temperature produced by laser heating. We increased the power up to a value where the laser caused a visible damage to the particle, $P \geq 15 \mathrm{~mW}$. The damage was signaled by the emission of microscopic polystyrene droplets, which could be seen drifting in the oil phase close to the magsphere. The laser induced dissolution presumably happens when the temperature inside the particle reaches about $100{ }^{\circ} \mathrm{C}$, which is close to the glass transition temperature of polystyrene. Supposing that $\Delta T$ $=T_{\text {mgs }}-T_{0}$, the difference between the average temperature $T_{\text {mgs }}$ of the magsphere and room temperature $T_{0}$, is proportional to $P$, we estimate $\Delta T \approx 5 P$ for the above laser beamwaist ( $P$ is in $\mathrm{mW}, \Delta T$ in $\mathrm{K})$. As a rough guide, the formula may be generalized to larger beamwaists as $\Delta T \approx$ $200\left(P / \omega_{0}^{2}\right)$, with $\omega_{0}$ in $\mu \mathrm{m}$ and $P$ in $\mathrm{mW}$. Then we estimate the temperature in Figure 6 as $\Delta T \approx 1.2 \mathrm{~K}$.

\section{DISCUSSION}

We start with a discussion about the forces at stake in the experiments, essentially "optical forces" associated with light momentum transfer, and hydrodynamic forces. Amplitudes of the optical forces are estimated based on a simplified representation of the particle internal structure.

Model for Particle Structure. (Figure 10a): we suppose a spherical PS core, surrounded by an absorbing spherical shell. The core, $a_{\mathrm{C}}$ in radius, is made of pure polystyrene (PS), of density $\rho_{\mathrm{PS}}$. The shell, $a_{\mathrm{S}}$ in outer radius, is a homogeneous mixture of PS and oxide. The density and volume fraction of the oxide are denoted as $\rho_{\mathrm{ox}}$ and $X$, respectively. The particle is in water, of density $\rho_{\mathrm{W}}$.

The sedimentation experiment (Section 3) allows us to determine the particle's buoyant weight $\bar{m} g=(4 / 3) \pi a_{S}^{3} \Delta \bar{\rho}$, and then its average density $\bar{\rho}=\Delta \bar{\rho}+\rho_{\mathrm{W}}$. We suppose that the density of the PS oxide mixture linearly interpolates between $\rho_{\mathrm{PS}}$, corresponding to $\mathrm{X}=0$, and $\rho_{\mathrm{ox}}$, corresponding to $\mathrm{X}=1$. The latter assumption leads to the simple relation:

$$
\varepsilon=\nu+\mu X(1-u)
$$

with

$$
\begin{gathered}
\varepsilon=\Delta \bar{\rho} /\left(\rho_{\mathrm{PS}}\right), \quad \nu=\left(\rho_{\mathrm{PS}}-\rho_{\mathrm{W}}\right) / \rho_{\mathrm{PS}}, \\
\mu=\left(\rho_{\mathrm{Ox}}-\rho_{\mathrm{PS}}\right) / \rho_{\mathrm{PS}}, \quad u=\left(a_{\mathrm{C}} / a_{\mathrm{S}}\right)^{3}
\end{gathered}
$$

From the microscope images, we estimate $a_{\mathrm{C}} \approx 1.10 \mu \mathrm{m}$ and $a_{\mathrm{S}} \approx 2.20 \mu \mathrm{m}$. The experiment with $\omega_{0}=3.65 \mu \mathrm{m}$ (Section 3) yields $\bar{m} g \approx 0.06 \mathrm{pN}$. We deduce $\Delta \bar{\rho} \approx 0.135 \mathrm{~g} \cdot \mathrm{cm}^{-3}$, within $\pm 7 \%$. With $\rho_{\mathrm{W}}=1.00, \rho_{\mathrm{PS}}=1.05$ and $\rho_{\text {ox }}=5.24 \mathrm{~g} \cdot \mathrm{cm}^{-3}$, we obtain $2.1 \% \leq X \leq 2.8 \%$. The latter value corresponds to about $9 \%$ for the oxide mass fraction within the particle.

Optical Forces. We want to determine the forces due to momentum transfer between the laser light and the particle. The refractive index of the core is simply that of polystyrene, $n$ $=n_{\mathrm{PS}}(=1.59)$ for $r \leq a_{\mathrm{C}}$. The oxide, which strongly absorbs light, has a complex index: $n_{\mathrm{ox}}=n_{\mathrm{ox}}^{\prime}+i n_{\mathrm{ox}}^{\prime \prime}$. Similarly to the density, we suppose that the shell index $n_{\mathrm{s}}=n^{\prime}+i n^{\prime \prime}$ linearly interpolates between $n_{\mathrm{PS}}$ and $n_{\mathrm{ox}}{ }^{27}$ Then: $n^{\prime}=(1-X) n_{\mathrm{PS}}+$ $X n_{\mathrm{ox}}^{\prime} ; n^{\prime \prime}=X n_{\mathrm{ox}}^{\prime \prime}$, for $a_{\mathrm{C}} \leq r \leq a_{\mathrm{S}}$. Numerical values for the oxide 

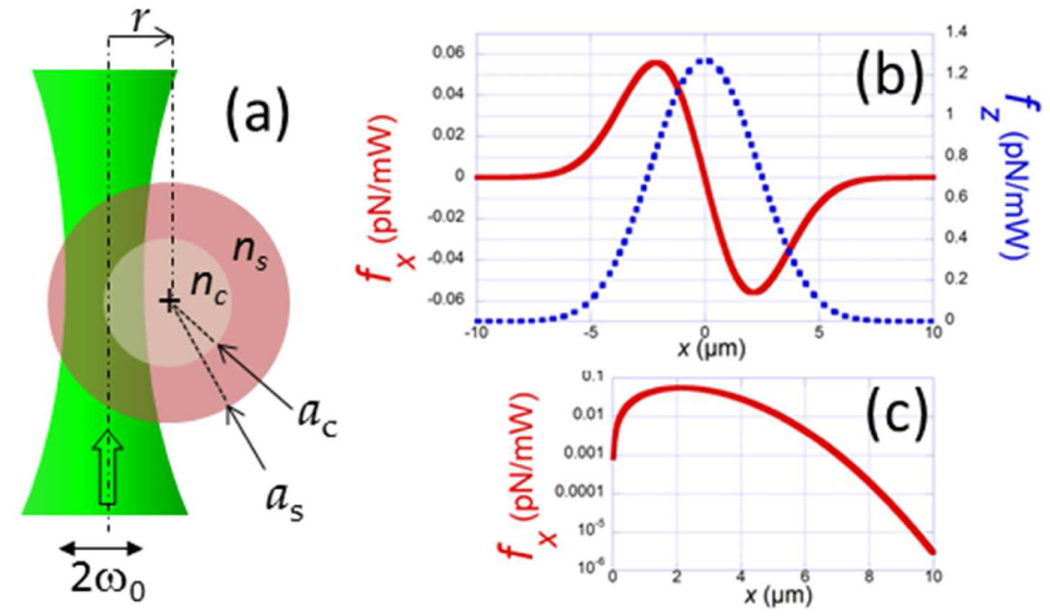

Figure 10. (a) Core-shell model for the magsphere. (b) Calculated profiles of the vertical (dotted line) and horizontal (solid line) components of the optical force for $\omega_{0}=3.65 \mu \mathrm{m}$. The beam is linearly polarized, perpendicularly to the figure. (c) Transverse force (in absolute value) versus $x$, in semilog representation, to help reading values of the force when the particle is far from the laser axis.

index are taken from the work by Buchenau and Müller: ${ }^{28} n_{\mathrm{ox}}^{\prime} \approx$ 2.20, $n_{\mathrm{ox}}^{\prime \prime} \approx 0.60$.

Optical Forces in Bulk Water. Calculation of the optical forces is based on the Generalized Lorenz-Mie Theory, using the ABSphere software. ${ }^{29}$ The theory supposes that the particle is immersed within an infinite homogeneous medium, and then can be directly used to simulate levitation experiments in bulk water. An example of calculated forces is shown in Figure 10b,c, for $\omega_{0}=3.65 \mu \mathrm{m}$, and $X=2.3 \%$. The graphs give the values of the axial (vertical) $f_{z}$ and transverse (horizontal) $f_{x}$ components in units of force per laser power $(\mathrm{pN} / \mathrm{mW})$, as functions of the particle position $x$. The corresponding forces are $F_{z}=f_{z} \cdot P$ and $F_{x}=f_{x} \cdot P$. The levitation force is maximum when the particle is centered on the laser axis: $f_{z, \max } \approx 0.13 \mathrm{pN} / \mathrm{mW}$. The transverse force is about linear around $x=0$, with a negative slope, meaning that the laser works as a spring that pulls the particle back in centered configuration. The laser beam then acts as a two dimensional trap, as observed in our experiments at small power. The corresponding stiffness constant is $k_{x} \approx 0.04$ $\mathrm{pN} \cdot \mu \mathrm{m}^{-1} \cdot \mathrm{mW}^{-1}$.

Knowing the buoyant weight of the particle, the calculation yields the theoretical value of the levitation power: $\bar{m} g=f_{z}(0) P_{\text {lev }}$. With $\bar{m} g \approx 0.06 \mathrm{pN}$, the simulation gives $P_{\text {lev }}$ $=0.035 \mathrm{~mW}$ for $\omega_{0}=3.65 \mu \mathrm{m}$, and $P_{\text {lev }}=0.15 \mathrm{~mW}$ for $\omega_{0}=$ $7.36 \mu \mathrm{m}$. The agreement is fairly good with the measured values, 0.045 and $0.20 \mathrm{~mW}$, respectively. We then suppose that the GLMT calculation based on the core-shell model for the particle structure can be used to estimate optical forces in general in the conditions of our experiments.

Optical Forces at the Water-Air Interface. Since GLMT is restricted to a particle inside an infinite homogeneous medium, the simulation cannot yield accurate results for the case of a particle sitting across the interface between two semi infinite media. Forces in bulk and at the interface cannot be the same. ${ }^{30}$ Nevertheless, we do not expect the difference between both situations to be considerable, insofar as we just want to obtain gross estimates of the optical force $F_{x}$ and compare them with-equally gross-estimates of hydrodynamic forces.

We saw that the laser would function as a $2 \mathrm{D}$ trap only at very small power $(P \sim 1 \mathrm{~mW})$ and that increasing $P$ would make the particle escape from the laser axis. Let us estimate the amplitude of the restoring optical force, which opposes the motion. In the conditions of Figure 4a, namely $\omega_{0}=6.30 \mu \mathrm{m}, P$ $=20 \mathrm{~mW}$, we find $f_{x, \max } P \sim 0.28 \mathrm{pN}$ at $x=3.3 \mu \mathrm{m}$. In the case of a wide beam as in Figure $3 \mathrm{~b}, \omega_{0}=33 \mu \mathrm{m}, P=36 \mathrm{~mW}, f_{x, \max } P$ $\sim 4 \times 10^{-3} \mathrm{pN}$ at $x=16 \mu \mathrm{m}$. Clearly, the restoring force is much less than $1 \mathrm{pN}$, in all experiments. Regarding the orbital motion, one might guess that $F_{x}$ is acting as the centripetal force that keeps the particle at finite distance, similarly to gravity acting on a satellite. Consider the example of Figure 10, for illustration. $r_{\text {orb }}$ is about $6 \mu \mathrm{m}$ for $P=10 \mathrm{~mW}$ (read Figure $5 \mathrm{a})$. In this case we estimate $f_{x}\left(r_{\text {orb }}\right) P \approx 0.04 \mathrm{pN}$, still a very small value.

Heat versus Momentum Transfer. Here we examine whether purely optical forces are large enough to make the particle move at velocities up to a few $100 \mu \mathrm{m} / \mathrm{s}$, as observed in the experiments. In other words, we ignore the effect of heat transfer and suppose that only the force due to light momentum transfer is at work. In this context, the optical force drives the particle through an otherwise quiescent fluid. In the purely viscous regime, the particle velocity is given by the balance between the optical force and the Stokes drag force: $F_{\mathrm{H}}$ $=6 \pi \eta a \nu$, where $\eta$ is the water viscosity $\left(\approx 10^{-3} \mathrm{~Pa} \cdot \mathrm{s}\right.$ at room temperature) and $\nu$ the particle velocity. The formula holds for a sphere in bulk water, but still can be used for the partially wetted particle as an order of magnitude estimate. ${ }^{31}$ With $\nu=$ $10 \mu \mathrm{m} / \mathrm{s}$, we estimate $F_{\mathrm{H}} \simeq 0.4 \mathrm{pN}$. In the conditions of Figure $3\left(\omega_{0}=33 \mu \mathrm{m}\right), F_{\mathrm{H}} \geq 1 \mathrm{pN}$, while the corresponding optical force is at least 2 orders of magnitude smaller $(\sim 0.004 \mathrm{pN}$, as we saw). The above estimates indicate that the optical force cannot have a real influence on the radial motion.

Optical forces cannot drive orbital motions either. At first sight, the configuration is axially symmetric around the laser beam axis; and the particle is spherically symmetric. Then the azimuthal component of the optical force should be null from symmetry. In reality, axial symmetry is not exactly realized, because the optical forces depend on the polarization of the laser beam. Another complication is the laser intensity distribution in the focal plane, which is slightly elliptical. However, both causes can only produce a very small azimuthal force, which reverses in sign every half period, and then cannot drive a continuous orbital motion.

If the particle departs from spherical symmetry, and simply has two different sides (vector symmetry), there may be a 
nonzero azimuthal component of the optical force $\left(F_{\theta}\right)$ that drives continuous orbital motion, as is the case in the experimental system of Mondal et al. $^{32}$ Indeed spherical symmetry of the magsphere is not exactly realized, due to shape imperfection and to the wetting configuration with a generally noncircular contact line. However, the role of optical forces can again be ruled out, based on quantitative arguments. For illustration, we come back to the case $\omega_{0}=3.65 \mu \mathrm{m}$, with $r_{\text {orb }} \approx 6 \mu \mathrm{m}, P=10 \mathrm{~mW}, f_{x, \max } \approx 0.6 \mathrm{pN}$ (Figure 10). At such a distance from the axis, the particle only intercepts a very small power, $P\left(r_{\text {orb }}\right) \sim 10^{-2} P$. A plausible scale for the optical force is $f_{x, \max } P\left(r_{\text {orb }}\right)$, which is about $0.006 \mathrm{pN}$. Presumably, $F_{\theta}$ cannot be larger than the latter estimate. Optical forces at such a distance from the axis are tiny, due to the very small power received by the particle. A typical orbital velocity around $100 \mu \mathrm{m} / \mathrm{s}$ corresponds to $F_{\mathrm{H}} \simeq 4 \mathrm{pN}$. To conclude, the optical force is negligibly small compared to the Stokes drag force.

The above discussion shows that light momentum transfer alone cannot be the mechanism that drives the particle motion. The power necessary to animate the system comes from heat transferred by the particle to the surrounding fluid. Indeed optical forces scale as $P / c$, where $c$ is the velocity of light, and the corresponding mechanical power scales as $P \cdot(\nu / c)$. The corresponding conversion efficiency is obviously very small $(\nu /$ $c \sim 10^{-13}$ ). Any process able to transform the heat generated by the particle's absorption into mechanical work is then likely to be much more efficient in driving particle's motion (see the discussion by Maggi et al. in the case of a light driven rotatory motion). ${ }^{33}$

Diffusive Heat Transfer and Thermo-capillary Flow. Most likely, the observed phenomena stem from the response of the interface to heat from the particle. The shape of the temperature profile around the particle in partial wetting configuration at WA interface is not known exactly. Never theless, a useful guide for reasoning is given by the solution of the problem for a uniform sphere inside the bulk fluid. The same solution holds at an interface between two fluids of equal thermal conductivities: ${ }^{34}$

$$
T(r)=T_{0}+\frac{Q}{2 \pi \Lambda} \frac{1}{r}=T_{0}+\Delta T^{(0)} \frac{a}{r}
$$

where $Q$ is the fraction of the laser power absorbed by the particle and dissipated into heat. Equation 4 is the solution to the heat diffusion equation, neglecting heat transfer by advection. $T_{0}$ is the temperature far from the particle, $\Lambda$ is the heat conductivity of the fluid $\left(\approx 0.6 \mathrm{~W} \cdot \mathrm{m}^{-1} \cdot \mathrm{K}^{-1}\right.$ for water $)$ and $r$ is the distance to the sphere center. $\Delta T^{(0)}=Q / 2 \pi \Lambda a$ is the temperature on the particle's surface. We expect the gradient in $T(r)$ to induce a gradient of the WA surface tension $\gamma:$

$$
\partial_{\mathrm{r}} \gamma(r)=\frac{\mathrm{d} \gamma}{\mathrm{d} T} \partial_{\mathrm{r}} T(r)
$$

Because the surface tension of water decreases with the temperature $\left(\mathrm{d} \gamma / \mathrm{d} T \simeq-0.144 \mathrm{mN} \cdot \mathrm{m}^{-1} \cdot \mathrm{K}^{-1}\right)$, the gradient in $\gamma$ around the hot particle induces a thermal Marangoni flow, or "thermocapillary" flow. Due to the spherical symmetry of the heat source, the flow has the shape of a torus, which is axially symmetric around the particle. ${ }^{34}$ The flow at the interface is centrifuge, with a velocity on the order of

$$
U_{\mathrm{Ma}}=\frac{\mathrm{d} \gamma}{\mathrm{d} T} \frac{\Delta T^{(0)}}{\eta}
$$

Putting numbers into eq 5 gives $U_{\mathrm{Ma}} \approx 10 \mathrm{~cm} / \mathrm{s}$ for $\Delta T^{(0)}=$ $1 \mathrm{~K}$, quite a huge value compared to experimental velocities. Whatever the amplitude of $U_{\mathrm{Ma}}$, surface tension forces around the spherical heat source should compensate due to the axial symmetry of the configuration, giving a zero net force. The sphere should then keep at rest. However, this equilibrium is very unstable, as it may be broken by any minute asymmetry of the temperature distribution. A "left-right" asymmetry gives a nonzero net force that propels the particle in some given direction, ${ }^{35}$ similarly to the case of microswimmers, which exploit a chemical Marangoni effect. ${ }^{36,37}$ The asymmetry may be simply dipolar (left-right), but may be more complex, including chiral shapes. The latter case has recently been exploited to make a hot particle rotate permanently around its axis. $^{33}$

The case of a dipolar asymmetry has been tackled in recent theoretical works (see refs 34 and 38). The particle is supposed to be hotter on one side, with a finite temperature difference between left and right. The difference may be caused by a nonuniform absorption in the particle volume when the particle is illuminated by a uniform laser beam. The amplitude of the asymmetry is measured by the $b / a$ ratio. The length $b$ corresponds to the offset of the temperature profile with respect to the particle center. The $b / a$ parameter is in general smaller than unity, and vanishes for a perfectly symmetric particle. The temperature dipole, through the Marangoni effect, propels the particle at velocity $\nu_{\mathrm{Ma}}$ given by

$$
v_{\mathrm{Ma}}=-\frac{1}{8} \frac{b}{a} U_{\mathrm{Ma}}
$$

Experimental velocities around $10 \mu \mathrm{m} / \mathrm{s}$ (see Figure 3) can then be accounted for by supposing a very small asymmetry, $2 b / a \sim 10^{-3}$. Such an asymmetry in the experimental system is very plausible in view of the imperfections of the magspheres, in shape, surface defects and distribution of the iron oxide inclusions. A related defect is the contact line configuration, which cannot be exactly circular. Irregularities in the contact line translate into irregular boundary conditions for heat diffusion and then are enough to produce asymmetries in the temperature field.

Most importantly, the velocity given by eq 6 is negative; which means that the particle moves in the direction opposite to the temperature gradient. In our experimental scheme, when the particle is off axis (the general situation), the inner side is at slightly higher temperature than the outer side. This difference causes a radially oriented dipolar term in the temperature distribution. The flow due to this dipole acts in pushing the particle off the laser axis.

Thermo-capillarity versus Concentration GradientDriven Flow. As we already mentioned, the presence of surface active contaminants is unavoidable in experiments with water-air interfaces. According to $\mathrm{Hu}$ and Larson, ${ }^{25,26}$ even an extremely small surface concentration $c_{\mathrm{S}} \sim 300$ molecules/ $(\mu \mathrm{m})^{2}$ is enough to reduce the amplitude of the thermo capillary flow by a factor of 100 . Because of this very high sensitivity, real surface velocities are much inferior to the theoretical value given by eq 5 .

The thermo capillary flow has the primary effect of conveying contaminants away from the heat source, and of accumulating them in the periphery of the free surface. Thus, a 
gradient in $c_{\mathrm{S}}$ is created, which in turn causes a gradient in the surface tension. Because the surface tension in general decreases with increasing $c_{S}$, the temperature and concentration gradients act in opposite directions. This simple argument is enough to explain why radial velocities are inferior to those expected with pure thermo convection.

Mizev and Trofimenko (MT) have studied the flow around a quasi point source at a water-air interface. ${ }^{39,40}$ The source might be a heated metal tip (see the 2005 paper by Mizev) or an alcohol-water solution that was injected just below the interface (2014 paper by Mizev and Trofimenko). In principle both types of sources have similar effects, since either heat or the concentration in alcohol tend to decrease the surface tension. The study was aimed at demonstrating the influence of contamination on the surface velocity field patterns. In the 2014 work, small amounts of oleic acid were spread on the water free surface to play the role of contaminants. Both the concentration of "surface contaminants" $c_{\mathrm{S}}$ and the rate $q$ of injected solution were controlled. The authors showed that the flow would self organize into a central zone, where the flow was simply radial and centrifuge, and, rather surprisingly, a peripheral zone, where the flow consisted of a number $N$ of vortices. MT's experimental data show that the vortices get larger and closer to the source when $c_{\mathrm{S}}$ increases. Conversely, increasing $q$ has the effect of increasing the size $r_{\mathrm{C}}$ of the radial velocity zone, and of decreasing the size of the vortices (concomitantly increasing $N$ ). We hereafter refer to such structures as "MT patterns". These patterns are produced by the competition between antagonist Marangoni effects, namely thermo capillarity (due to the temperature gradient) and chemo capillarity (due to the gradient in $c_{S}$ ). In their tentative representation of the coupling between the flow and $c_{S}$, MT imagine that the contaminants have been swept out of the central zone $\left(r \geq r_{\mathrm{C}}\right)$ (see Figure 6 of the 2014 paper).

The behaviors of laser heated particles most probably share characteristics with the above described MT patterns. We assume that switching on the laser initially has the effect of repelling contaminants from the hot particle up to a distance $r_{\mathrm{C}}$ and that the flow beyond $r_{\mathrm{C}}$ self organizes into vortices, similarly to MT patterns.

The main difference between our experiments and those of MT is that the heated particle is free to move on the surface, contrary to MT's metal tip or injector. The freedom given to the particle leads to the orbits (and confined trajectories, in general) that we have described. Since MT patterns and hot particle trajectories stem from the same physical ingredients, we believe that orbit radii are about the same as $r_{\mathrm{C}}$. To our knowledge, MT patterns have not been accounted for by any theoretical model, presumably because of the complexity of the problem. Elaborating such a model is necessary to predict how $r_{\mathrm{C}}$ (and the orbit size, hopefully), depend on the system parameters $\left(P, \omega_{0}, a\right)$. The latter question calls for future developments of the theory.

Evaporation versus Thermo-capillary Flow. The hot magsphere is an experimental realization of a heat source close to a contact line (CL). Our system has similarities with experiments involving menisci in studies dedicated to evaporation. $^{25,26,41,42}$ For instance, Sefiane and Ward have studied the influence of a heat source around the meniscus of a liquid in a cylindrical tube. ${ }^{41}$ They clearly observed convection away from CL when the heat source was strong enough (see Figure 36 in ref 41), similarly to what happens around the hot sphere (our Figure 6). In parallel to the Marangoni effect, evaporation is certainly important close to CL. As we explained, tracers accumulate along CL under laser illumination, and get released when the laser is off. In some experiments, the tracers are conveyed by an outward convective flow when the laser is switched off (Figure 8). Presumably these facts can only be explained as due to evaporation (laser on) and vapor condensation following laser switch off. Condensation is what we believe to be the source of the centrifuge flow (laser off) in Figure 8.

We thus arrive at the general scheme of Figure 7, where evaporation drives a centripetal flow (laser on) close to the particle, and thermo capillarity drives a centrifuge flow beyond $d_{\text {min }}$. In this view, the centripetal flow is analogous to what causes the coffee ring effect with an evaporating water sessile drop. The flow conveys fluid as a response to the loss of liquid due to evaporation. Because the evaporation rate is highest (and even diverges) close to $\mathrm{CL},{ }^{43}$ the flow is directed toward the particle.

In the stationary regime, the mass flux of water taken by evaporation must be compensated by an inward flux of liquid water toward the particle, as suggested in Figure 7. The zone directly above and around the particle is certainly highly concentrated in hot water vapor. The resulting gradients in concentration, temperature, and pressure expectably drive a radial mass transfer of the vapor, resulting in recondensation at some distance from the axis. Recondensation thus provides the feedback loop for the inward liquid water flow to be permanent. We currently work on elaborating a quantitative description of this process.

Fluctuations of Orbital Velocity. The fact that orbital velocities $\nu_{\text {orb }}$ take on apparently random values, including intermittence and sign reversal within a given experiment, may seem mysterious. Orbiting at constant distance from the laser axis means that the particle lies in a radial temperature gradient. $Q$ and $\partial_{\mathrm{r}} Q$ are constant along the orbit. The motion is only possible at the expense of symmetry breaking in the azimuthal direction $(\theta)$, equivalently to what was quantified by $2 b / a$ in the dipolar model for the radial motion. The variations in $\nu_{\text {orb }}$ indicate that the amplitude of $(2 b / a)_{\theta}$ is not constant over time periods of the order of minutes. The latter quantity may then fluctuate, and even reverse in sign, due to thermally activated changes in the configuration of the contact line. In this view, the evolution of the contact line is analogous to the process described by Kaz et al. for a system at uniform temperature. ${ }^{20}$ The process with the hot particle is certainly much faster (on the scale of seconds) than with the same system but at uniform (room) temperature.

From Magspheres to Other Types of Particles. We end this section with a remark about the generality of the observed particle motions. We only reported data obtained with magspheres, a particular type of particle whose choice was dictated by resistance to photobleaching and overall structural stability. We carried out a few extra experiments with bitumen emulsion droplets. ${ }^{44}$ These droplets have the advantage of being made of a uniform strongly absorbing internal material and look spherical in microscope images. Such droplets could be easily levitated and brought to the WA interface. Experiments carried out with a broad beam $\left(\omega_{0}=33 \mu \mathrm{m}\right)$ showed off axis motions very similar to those observed with magspheres (Figure 3). The drawback of bitumen droplets is that, once at the interface, they only survive for a minute or so, presumably due to the spreading of the droplet material on the interface. Since the emulsion contains surface active species, the 
interface gets contaminated on the scale of a minute. Nevertheless, the observed responses of the droplets convinced us that behaviors observed with magspheres were not specific of their particular structure.

\section{CONCLUSION}

Using a visible laser and light absorbing particles, we have studied the behavior of a laser heated spherical particle sitting in partial wetting at the interface between water and air. We showed that this apparently very simple system, a hot particle across the interface, behaved in surprising ways, transiting from stably trapped by the laser at small power to "laser repelled" at higher powers. We even observed that the particle would orbit around the laser axis at very high velocities, up to almost $1 \mathrm{~mm} /$ $\mathrm{s}$ with just a few milliwatts of laser power. Radii of the orbits were stable and reproducible, but rather intriguingly, we observed that orbital velocities seemed random. It was not rare that a particle orbit would reverse in direction in the course of a single experiment.

Observations of the flow around the hot particle with small tracers revealed that both thermo capillarity and evaporation were active, with evaporation presumably dominant close to the particle-water-air contact line.

A theory of the observed phenomena is still lacking. We could just rule out simplistic interpretations based on optical forces, whose amplitudes turn out too small to explain the measured velocities. Clearly the hot particle is propelled by flows originating from the heat transferred from the particle body to the fluid. The latter conclusion is not really surprising. This is just one illustration of the general principle according to which heat transfer from light to the particle can be much more efficient than direct momentum transfer to produce mechanical work. $^{33}$

We proposed a few tentative explanations for different aspects of the laser heated particle's motion. Thermo capillarity is certainly the dominant mechanism that destabilizes optical trapping and drives the particle off the laser axis. The particle's orbital motion seems to stem from the competition between two antagonist Marangoni effects, due to the temperature gradient and the presence of surface active contaminants. The latter proposition was inspired by similarities between flow structures observed by Mizev and Trofimenko with fixed sources, ${ }^{39,40}$ and our observations, with a mobile source. We presume that fluctuations in the particle velocity are mainly due to fluctuations of the contact line, which are evident in observations. Evaporation comes in as a further mechanism; but its influence and possible competition with Marangoni driven flows is not yet elucidated. Further understanding calls for experimental and theoretical developments complemented by numerical simulations. These works are currently in progress.

\section{AUTHOR INFORMATION}

\section{Corresponding Author}

*E mail: pouligny@crpp bordeaux.cnrs.fr.

Notes

The authors declare no competing financial interest.

\section{ACKNOWLEDGMENTS}

CRPP and CORIA have received funding from Agence Nationale de la Recherche (ANR) under "AMOCOPS” project (ANR 13 BS09 008). LOMA has received funding from ANR under "HOTSPOT" project (ANR-13-IS04-0003). LOMA and CRPP have received funding from IDEX-Bordeaux under "Propulsion de micro-nageurs par effet Marangoni” PEPS program. The authors are grateful to F. Nadal, J.B. Salmon, Th. Fischer, P. Kralchevsky for fruitful discussions. We thank P. Snabre for his help with particle-tracking software, V. Schmitt and P. Anaclet for the kind provision of bitumen emulsion samples, I. Ly, CRPP instrumentation and mechanic groups for technical help.

\section{REFERENCES}

(1) Ashkin, A. Acceleration and trapping of particles by radiation pressure. Phys. Rev. Lett. 1970, 24, 156-159.

(2) Rodrigo, P. J.; Daria, V. R.; Glückstad, J. Real time three dimensional optical micromanipulation of multiple particles and living cell. Opt. Lett. 2004, 29, 2270-2272.

(3) Rodrigo, P. J.; Gammelgaard, L.; Bøggild, P.; Perch Nielsen, I.; Glückstad, J. Actuation of microfabricated tools using multiple GPC based counterpropagating beam traps. Opt. Express 2005, 13, 68996904.

(4) Rodrigo, P. J.; Daria, V.; Glückstad, J. Four dimensional optical manipulation of colloidal particles. Appl. Phys. Lett. 2005, 86, 074103.

(5) Mihiretie, B. M.; Snabre, P.; Loudet, J. C.; Pouligny, B. Radiation pressure makes ellipsoidal particles waltz. Europhys. Lett. 2012, 100, 48005 .

(6) Mihiretie, B. M.; Snabre, P.; Loudet, J. C.; Pouligny, B. Optically driven oscillations of ellipsoid particles. Part I: Experimental observations. Eur. Phys. J. E: Soft Matter Biol. Phys. 2014, 37, 124.

(7) Ashkin, A.; Dziedzic, J. M.; Bjorkholm, J. E.; Chu, S. Observation of a single beam gradient force optical trap for dielectric particles. Opt. Lett. 1986, 11, 288-290.

(8) Chan, D. Y. C.; Henry, J. D.; White, L. R. The interaction of colloidal particles collected at fluid interfaces. J. Colloid Interface Sci. 1981, 79, 410-418.

(9) In principle, $2 \mathrm{~d}$ trapping of a very absorbing sphere may be achieved with a strongly converging Gaussian beam. A hollow beam $\left(\mathrm{TEM} 01^{*}\right)$ is a simpler and more efficient alternative. See Rubinsztein Dunlop, H.; Nieminen, T. A.; Friese, M. E. J.; Heckenberg, N. R. Optical trapping of absorbing particles. Adv. Quantum Chem. 1998, 30, 469-492.

(10) Aliaskarisohi, S. M.; Fischer, T.; Wilke, N. Dilatational Yielding of Solid Langmuir Monolayers. J. Phys. Chem. B 2011, 115, 1163111637.

(11) Muruganathan, R. M.; Khattari, Z.; Fischer, T. M. Non equilibrium Bubbles in a Flowing Langmuir Monolayer. J. Phys. Chem. B 2005, 109, 21772-21778.

(12) Muruganathan, R. M.; Fischer, T. M. Laser Induced Local Collapse in a Langmuir Monolayer. J. Phys. Chem. B 2006, 110, 22160-22165.

(13) Gugliotti, M.; Baptista, M. S.; Politi, M. J. Laser Induced Marangoni Convection in the Presence of Surfactant Monolayers. Langmuir 2002, 18, 9792-9798.

(14) Varanakkottu, S. N.; George, S. D.; Baier, T.; Hardt, S.; Ewald, M.; Biesalski, M. Particle Manipulation Based on Optically Controlled Free Surface Hydrodynamics. Angew. Chem., Int. Ed. 2013, 52, 72917295.

(15) Diguet, A.; Guillermic, R. M.; Magome, N.; Saint Jalmes, A.; Chen, Y.; Yoshikawa, K.; Baigl, D. Photomanipulation of a Droplet by the Chromocapillary Effect. Angew. Chem., Int. Ed. 2009, 48, 92819284.

(16) Lauga, E.; Powers, T. R. The hydrodynamics of swimming microorganisms. Rep. Prog. Phys. 2009, 72, 096601. 
(17) Mihiretie, B. M. Mechanical effect of light on anisotropic micron sized particles and their wetting dynamics at the water air interface. Ph.D. Thesis, Université Bordeaux 1, July 2013.

(18) Stamou, D. Long range attraction between colloidal spheres at the air water interface: The consequence of an irregular meniscus. Phys. Rev. E: Stat. Phys., Plasmas, Fluids, Relat. Interdiscip. Top. 2000, 62, 5263-5272.

(19) Chen, W.; Tan, S.; Ng, T. K.; Ford, W. T.; Tong, P. Long ranged attraction between charged polystyrene spheres at aqueous interfaces. Phys. Rev. Lett. 2005, 95, 21830.

(20) Kaz, D. M.; McGorty, R.; Mani, M.; Brenner, M. P.; Manoharan, V. N. Physical ageing of the contact line on colloidal particles at liquid interfaces. Nat. Mater. 2012, 11, 138-142.

(21) Nadal, F.; Dazzi, A.; Argoul, F.; Pouligny, B. Probing the dynamics of a spherical colloid close to a surface by combined optical trapping and reflection interference contrast. Appl. Phys. Lett. 2001, 79, 3887-3889.

(22) Roosen, G.; Imbert, C. Optical levitation by means of two horizontal laser beams: A theoretical and experimental study. Phys. Lett. A 1976, 59, 6-8.

(23) Roosen, G. A theoretical and experimental study of the stable equilibrium positions of spheres levitated by two horizontal laser beams. Opt. Commun. 1977, 21, 189-194.

(24) Saylor, J. R.; Smith, G. B.; Flack, K. A. Infrared imaging of the surface temperature field of water during film spreading. Phys. Fluids 2000, 12, 597-602.

(25) Hu, H.; Larson, R. G. Analysis of the Effects of Marangoni Stresses on the microflow in an Evaporating Sessile Droplet. Langmuir 2005, 21, 3972-3980.

(26) Hu, H.; Larson, R. G. Marangoni Effect Reverses Coffee Ring Depositions. J. Phys. Chem. B 2006, 110, 7090-7094.

(27) Heller, W. Remarks on Refractive Index Mixture Rules. J. Phys. Chem. 1965, 69, 1123-1129 The simple linear superposition of indices, known as Biot's formula, was chosen for simplicity. Since the iron oxide is not homogeneously distributed within the PS shell (see Figure 2a), it is not really important to make a choice between models which all of them are restricted to homogeneous mixtures. Using e.g. the Lorentz Lorenz formula leads to slightly different values of the effective complex index but does not make a significant difference in values of the calculated levitation force..

(28) Buchenau, U.; Müller, I. Optical properties of magnetite. Solid State Commun. 1972, 11, 1291-1293.

(29) Ren, K. F.; Gréhan, G.; Gouesbet, G. Radiation pressure forces exerted on a particle arbitrarily located in a Gaussian beam by using the generalized Lorenz Mie theory, and associated resonance effects.
Opt. Commun. 1994, 108, 343-354. The ABSphere software can be freely downloaded from https://amocops.univ rouen.fr/en/content/ download.

(30) Park, B. J.; Furst, E. M. Optical trapping forces for colloids at the oil water interface. Langmuir 2008, 24, 13383-13392.

(31) Danov, K.; Aust, R.; Durst, F.; Lange, U. Influence of the Surface Viscosity on the Hydrodynamic Resistance and Surface Diffusivity of a Large Brownian Particle. J. Colloid Interface Sci. 1995, $175,36-45$

(32) Mondal, A.; Roy, B.; Banerjee, A. Generation of microswimmers from passive Brownian particles in a spherically aberrated optical trap. Opt. Express 2015, 23, 8021-8028.

(33) Maggi, C.; Saglimbeni, F.; Dipalo, M.; De Angelis, F.; Di Leonardo, R. Micromotors with asymmetric shape that efficiently convert light into work by thermocapillary effects. Nat. Commun. 2015, 6, 7855 .

(34) Würger, A. Thermally driven Marangoni surfers. J. Fluid Mech. 2014, 752, 589-601.

(35) Wang, X.; In, M.; Blanc, C.; Nobili, M.; Stocco, A. Enhanced active motion of Janus colloids at the water surface. Soft Matter 2015, $11,7376-7384$

(36) Nakata, S.; Iguchi, Y.; Ose, S.; Kuboyama, M.; Ishii, T.; Yoshikawa, K. Self Rotation of a Camphor Scraping on Water: New Insight into the Old Problem. Langmuir 1997, 13, 4454-4458.

(37) Lauga, E.; Davis, A. M. J. Viscous Marangoni propulsion. J. Fluid Mech. 2012, 705, 120-133.

(38) Masoud, H.; Stone, H. A. A reciprocal theorem for Marangoni propulsion. J. Fluid Mech. 2014, 741, R4 1-R4 7.

(39) Mizev, A. Influence of an adsorption layer on the structure and stability of surface tension driven flows. Phys. Fluids 2005, 17, 122107.

(40) Mizev, A.; Trofimenko, A. I. Effect of an Insoluble Surfactant Film on the Stability of the Concentration Driven Marangoni Flow. Fluid Dyn. 2014, 49, 26-36.

(41) Sefiane, K.; Ward, C. A. Recent advances on thermocapillary flows and interfacial conditions during the evaporation of liquids. Adv. Colloid Interface Sci. 2007, 134-135, 201-223.

(42) Deegan, R. D.; Bakajin, O.; Dupont, T. F.; Huber, G.; Nagel, S. R.; Witten, T. A. Capillary flow as the cause of ring stains from dried liquid drops. Nature 1997, 389, 827-829.

(43) Cazabat, A. M.; Guéna, G. Evaporation of macroscopic sessile droplets. Soft Matter 2010, 6, 2591-2612.

(44) Boucard, L.; Schmitt, V.; Farcas, F.; Gaudefroy, V. Bitumen emulsions formulation and destabilisation process relationship: influence of salts addition. Road Mater. Pavement Des. 2015, 16, $330-348$. 